\title{
The Therapeutic Effect of Second Near-Infrared Absorbing Gold Nanorods on Metastatic Lymph Nodes via Lymphatic Delivery System
}

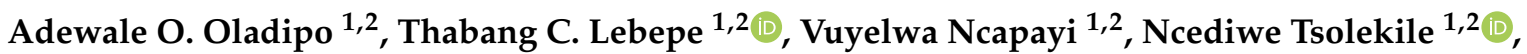 \\ Sundararajan Parani ${ }^{1,2} \mathbb{D}$, Sandile P. Songca ${ }^{3} \mathbb{D}$, Shiro Mori ${ }^{4,5}$, Tetsuya Kodama ${ }^{4, *} \mathbb{D}$ \\ and Oluwatobi S. Oluwafemi $1,2, *$ [D
}

check for updates

Citation: Oladipo, A.O.; Lebepe, T.C.; Ncapayi, V.; Tsolekile, N.; Parani, S.; Songca, S.P.; Mori, S.; Kodama, T.; Oluwafemi, O.S. The Therapeutic Effect of Second Near-Infrared Absorbing Gold Nanorods on Metastatic Lymph Nodes via Lymphatic Delivery System. Pharmaceutics 2021, 13, 1359. https://doi.org/10.3390/ pharmaceutics13091359

Academic Editors: Matt Kipper and Alessandro F. Martins

Received: 5 July 2021

Accepted: 10 August 2021

Published: 28 August 2021

Publisher's Note: MDPI stays neutral with regard to jurisdictional claims in published maps and institutional affiliations.

Copyright: (c) 2021 by the authors. Licensee MDPI, Basel, Switzerland. This article is an open access article distributed under the terms and conditions of the Creative Commons Attribution (CC BY) license (https:/ / creativecommons.org/licenses/by/ $4.0 /)$.
1 Department of Chemical Sciences, University of Johannesburg Doornfontein Campus, Johannesburg 2028, South Africa; 201504949@student.uj.ac.za (A.O.O.); tlebepe@uj.ac.za (T.C.L.); 216087577@student.uj.ac.za (V.N.); 201504952@student.uj.ac.za (N.T.); PSundararajan@uj.ac.za (S.P.)

2 Centre for Nanomaterials Science Research, University of Johannesburg, Johannesburg 2028, South Africa

3 Department of Chemistry, University of KwaZulu-Natal, Private Bag X 54001, Durban 4000, South Africa; songcaS@ukzn.ac.za

4 Graduate School of Biomedical Engineering, Tohoku University, 4-1 Seiryo, Aoba, Sendai 980-8575, Japan; Shiro.mori.a5@tohoku.ac.jp

5 Department of Oral and Maxillofacial Surgery, Tohoku University Hospital, 1-1 Seiryo, Aoba, Sendai 980-8575, Japan

* Correspondence: kodama@tohoku.ac.jp (T.K.); oluwafemi.oluwatobi@gmail.com (O.S.O.)

\begin{abstract}
Photothermal therapy has been established recently as a non-invasive treatment protocol for cancer metastatic lymph nodes. Although this treatment approach shows efficient tumour ablation towards lymph node metastasis, the monitoring and reporting of treatment progress using the lymphatic delivery channel still need to be explored. Herein, we investigated the anti-tumour effect of pegylated gold nanorods with a high aspect ratio (PAuNRs) delivered via the lymphatic route in a mouse model. In this study, breast carcinoma (FM3A-Luc) cells were inoculated in the subiliac lymph node (SiLN) to induce metastasis in the proper axillary lymph node (PALN). The treatment was initiated by injecting the PAuNRs into the accessory axillary lymph node (AALN) after tumour metastasis was confirmed in the PALN followed by external NIR laser irradiation under a temperature-controlled cooling system. The anti-tumour impact of the treatment was evaluated using an in vivo bioluminescence imaging system (IVIS). The results showed a time-dependent reduction in tumour activity with significant treatment response. Tumour growth was inhibited in all mice treated with PAuNRs under laser irradiation; results were statistically significant $(* * p<0.01)$ even after treatment was concluded on day 3. We believe that this non-invasive technique would provide more information on the dynamics of tumour therapy using the lymphatically administered route in preclinical studies.
\end{abstract}

Keywords: lymphatic route; gold nanorods; photothermal therapy; metastatic lymph node; nearinfrared light

\section{Introduction}

Cancer remains one of the leading causes of mortality worldwide, and most cases are due to lymph node (LN) metastasis [1]. To date, many measures have been developed to combat this trend, with remarkable therapeutic outcomes [2-5]; however, there is still a growing number of physical and emotional side effects associated with these protocols [6,7]. The destruction of tumour metastasis under hyperthermia has been investigated in the past few years as a non-invasive treatment method [8,9]. For deep-embedded metastatic LN photothermal therapy, the use of gold nanorods with a large cross-section absorption in the second biological NIR window (900-1100) is required [3,10-12]. Gold-nanorods' 
(AuNRs) unique shape-dependent longitudinal surface plasmon resonance (LSPR) and their optical properties make them an ideal nanoparticle for the laser-induced destruction of tumour cells [13-15]. In addition, their ease of functionalisation with targeting ligands for maximum accumulation in the tumour matrix $[16,17]$, biocompatibility for a longterm in vivo existence [18-20], and decreased reticuloendothelial clearance [21] make them ideal hyperthermia candidates. Under laser light irradiation, AuNRs absorb light, which is converted into heat, resulting in hyperthermia-induced cell death and subsequent tumour regression [9,22-24]. The delivery of anti-cancer agents to metastatic sites is usually achieved through intravenous administration $[25,26]$. However, only a fraction of the administered drug dosage can reach the target site due to the preferential reabsorption of small anti-cancer drug molecules into blood capillaries $[5,27,28]$. This often results in insufficient dosage accumulation and retention of the anti-cancer agents required to treat metastasis effectively. In addressing this issue, our group reported a more efficient method for anti-cancer drug delivery to metastatic sites via the lymphatic route $[24,29,30]$. This delivery route has motivated the design of new nanocomposite-based therapeutic approaches as alternatives to invasive treatment protocols [31,32]. While this delivery route resulted in sufficient dosage delivery to target sites in high concentrations and with effective tumour cell destruction and growth termination under external NIR laser irradiation, little is known about the therapeutic dynamics and treatment prediction.

In the present study, the triangular network of lymph nodes linked together by the lymphatic vessels was used to evaluate and quantitatively monitor the therapeutic opportunities of lymphatically delivered, high-aspect-ratio AuNRs for photothermal applications in a mouse model [33]. NIR laser irradiation at the metastatic lymph node site commenced $160 \mathrm{~s}$ after lymphatic administration, with sufficient accumulation in the tumour area within a short time. To assess the antiangiogenic treatment effect and tumour growth luciferase expression, an in vivo bioluminescence imaging system (IVIS) was used. Our results indicate that tumour ablation and growth inhibition using this delivery route is a function of treatment time. This procedure can provide extensive information for the precise control of treatment in preclinical trials.

\section{Materials and Methods}

\subsection{Materials}

Hydrogen tetrachloroaurate hydrate (99.9\%), $\mathrm{NaBH}_{4}(99 \%)$, silver nitrate $(99 \%), \mathrm{CTAB}$ ( $\geq 99 \%)$, ascorbic acid $(99 \%)$, and $\mathrm{NaOL}(\geq 99 \%)$ were purchased from Sigma-Aldrich (Kempton Park, South Africa). Hydrochloric acid (ACS reagent, $37 \mathrm{wt} \%$ ) was obtained from Wako (Osaka, Japan). All chemicals were of analytical grade and were used without further purification. All solutions, other than gold and CTAB, were freshly prepared. The glassware used in the experiments was cleaned and washed thoroughly with MilliQ water and dried before use. A Millipore UV-synergy water purification system of $18.2 \mathrm{M} \Omega \mathrm{cm} @$ $25^{\circ} \mathrm{C}$ resistivity was used for water production.

\subsection{PEGylated Gold Nanorod Synthesis}

AuNRs were synthesised using a seed-mediated technique adapted from Ye et al. [34], with slight modifications. In a typical synthesis procedure, the seed solution was prepared by adding $0.3645 \mathrm{~g}$ of $\mathrm{CTAB}$ to $10 \mathrm{~mL} 40^{\circ} \mathrm{C}$ warm water. The solution was allowed to cool to room temperature before adding $0.250 \mathrm{~mL}$ of $\mathrm{HAuCl}_{4} \mathrm{xH}_{2} \mathrm{O}$ solution $(0.01 \mathrm{M})$ followed by stirring the mixture for $30 \mathrm{~min}$. A freshly prepared solution of $0.6 \mathrm{~mL}$ of $0.01 \mathrm{M} \mathrm{NaBH} 4$ was added to this solution under vigorous stirring for $2 \mathrm{~min}$ to produce a light brown solution that served as the seed solution. The solution was kept at $30^{\circ} \mathrm{C}$ for $30 \mathrm{~min}$ before use. In a $100 \mathrm{~mL}$ Erlenmeyer flask, $0.037 \mathrm{M}$ of CTAB and $0.1234 \mathrm{~g} \mathrm{NaOL}$ were dissolved in $25 \mathrm{~mL}$ of warm water $\left(\sim 40^{\circ} \mathrm{C}\right)$ to prepare the growth solution. The solution was allowed to cool down to $30^{\circ} \mathrm{C}$, and $1.2 \mathrm{~mL}$ of $\mathrm{AgNO}_{3}(4 \mathrm{mM})$ solution was added, followed by gentle stirring for $15 \mathrm{~min}$. To this solution, $1 \mathrm{mM} \mathrm{HAuCl} 4 \cdot \mathrm{H}_{3} \mathrm{O}$ solution was added, and the solution was further stirred for $1 \mathrm{~h}$, followed by $\mathrm{pH}$ adjustment by adding $0.96 \mathrm{~mL}$ 
of $37 \% \mathrm{HCl}$. After stirring for $15 \mathrm{~min}, 1.25 \mathrm{~mL}$ of ascorbic acid $(0.064 \mathrm{M})$ was added, and the solution was vigorously stirred for $30 \mathrm{~s}$. After this, $4 \mu \mathrm{L}$ of seed solution was added to the growth solution, and the resultant mixture was stirred for $30 \mathrm{~s}$ and left undisturbed at $30^{\circ} \mathrm{C}$ for $12 \mathrm{~h}$. The AuNR solution was centrifuged at $7000 \mathrm{rpm}$ for $30 \mathrm{~min}$ to remove excess unreacted reagents. AuNR ligand exchange was achieved by mixing $500 \mu \mathrm{L}$ of AuNRs with $500 \mu \mathrm{L}$ of $1 \mathrm{mM}$ mPEG-SH (MW 5000) and $100 \mu \mathrm{L}$ 1X-PBS under magnetic stirring for $12 \mathrm{~h}$. The final mPEG-SH functionalised AuNRs (PAuNRs) were centrifuged to remove any unbound $\mathrm{mPEG}-\mathrm{SH}$ and sonicated for $10 \mathrm{~min}$. The nanorods were redispersed in 1X-PBS solution for further use.

\subsection{Characterisation of AuNRs}

Absorption spectra were primarily obtained using a JASCO V-770 NIR spectrophotometer. TEM was performed using a JEOL JEM-2100F HRTEM with accelerating voltage of $200 \mathrm{kV}$. Surface charge measurements were performed using a Photal ELS-Z2MH instrument. Au content in tissues was determined using a PerkinElmer Optima 8300 ICP-OES Spectrometer (Perkin-Elmer, Waltham, MA, USA).

\subsection{Mice}

The mice used in these experiments (2013BeA-019 \& 2016BeA-004) were MXH10/Molpr/lpr [33], bred under specific pathogen-free conditions at the Animal Research Institute, Graduate School of Biomedical Engineering, Tohoku University, Sendai, Miyagi, Japan. They had unique LNs, growing up to $10 \mathrm{~mm}$ in size, equivalent to humans at $2.5-3$ months of age. In addition, these mice do not develop severe autoimmune diseases.

\subsection{FM3A-Luc Cell Culture}

RPMI-1640 medium (Sigma-Aldrich) (supplemented with 10\% heat-inactivated foetal bovine serum, 0.5\% Geneticin G418, and 1\% L-glutamine-penicillin-streptomycin (SigmaAldrich)) was used to cultivate FM3A-Luc (mouse mammary carcinoma cells containing luciferase gene expression) cells, in an incubator under the conditions of $37^{\circ} \mathrm{C}, 5 \% \mathrm{CO}_{2}$, and $95 \%$ air to achieve $80 \%$ confluence [24]. The cells were evaluated using a MycoAlert Mycoplasma Detection Kit (Lonza Rockland, Inc, Rockland, ME, USA) before use for the absence of Mycoplasma contamination, following the manufacturer's protocol.

\subsection{Metastasis Induction of PALN with Tumour Cells from the SiLN}

Metastasis was induced in PALN by injecting a suspension of $3.3 \times 10^{5}$ cells in $20 \mu \mathrm{L}$ PBS and $40 \mu \mathrm{L}$ of $400 \mathrm{mg} / \mathrm{mL}$ Matrigel (Collaborative Biomedical Products, Bedford, MA, USA) into the unilateral SiLN ( $n=24)$ [35]. Briefly, a $27 \mathrm{G}$ needle was used to carry out the intranodal inoculation into the SiLN with the assistance of a high-frequency ultrasound imaging system (VEVO770; VisualSonics Fuji Film, Tokyo, Japan) with a $25 \mathrm{MHz}$ transducer (RMV-710B; axial resolution, $70 \mu \mathrm{m}$; focal length, $15 \mathrm{~mm}$; VisualSonics FujiFilm). Matrigel solidification after removing the needle was achieved by maintaining the needle in the same position for one minute. The inoculation day was marked as day 0 . The metastasis to the PALN was evaluated every three days post-inoculation using an in vivo bioluminescence imaging system (IVIS Lumina; PerkinElmer, Waltham, MA, USA), with a background luciferase activity of $\sim 4 \times 10^{4}$ photons/s. Metastatic mice were considered when the luciferase activity in the PALN was greater than $\sim 5 \times 10^{5}$ photons $/ \mathrm{s}$. Luciferin $(150 \mathrm{mg} / \mathrm{kg}$, Promega Co., Madison, WI, USA) was injected intraperitoneally to measure luciferase bioluminescence after $10 \mathrm{~min}$ for $1 \mathrm{~min}$, using IVIS. The metastatic mice were divided into four groups: Control group (group I), PAuNRs group (group II), Laser group (group III), and PAuNRs + Laser group (group IV). Each group contained five mice. The highfrequency ultrasound system (VEVO770; VisualSonics) was used to measure the PALN volume changes on day 0 and day 18. 


\subsection{In Vivo Evaluation of the Metastatic PALNs Treated with PAuNRs and Laser}

In vivo photothermal treatment of PALNs was performed using laser irradiation after injection with PAuNRs. In short, the metastatic group IV mice were intranodally injected with PAuNRs $(40 \mu \mathrm{g} / \mathrm{mL}, 120 \mu \mathrm{L})$ into the AALNs. After $160 \mathrm{~s}$ post-injection, the temperature control system (cooling system water temperature, $10^{\circ} \mathrm{C}$; water flow rate, $760 \mathrm{~mL} / \mathrm{s}$ ) was used to cool down the skin temperature to $<15^{\circ} \mathrm{C}$ before irradiation. The mice PALNs were irradiated with a continuous-wave $\mathrm{Nd}_{\mathrm{YVO}} \mathrm{YV}_{4}$ air-cooled NIR laser $\left(2.5 \pm 0.5 \mathrm{~W} / \mathrm{cm}^{2}\right.$; wavelength, $1064 \mathrm{~nm}$; TEM 00 beam diameter, $0.6 \mathrm{~mm}$; CYD-010-TUBC, Neoarc) with a laser focus diameter of up to $0.6 \mathrm{~cm}$. The PALN's temperature was maintained at $45^{\circ} \mathrm{C}$ for 5 min by tuning the intensity of the laser beam. A K-type thermocouple (Ishikawa Trading Co. Ltd., Tokyo, Japan) was used to measure the site temperature during irradiation. The PAuNRs' anti-tumour efficacies were evaluated by measuring the luciferase activities in the metastasised PALN on day $0,1,2,3,4$, and 6 after treatment, using IVIS Lumina. The first day of treatment was marked as day zero. Aluminium foil and black cardboard were used to obscure the SiLNs in order to monitor the luciferase activity in the PALNs effectively. The luciferase intensity covered by the region of interest (ROI) around the PALNs was measured.

\subsection{PAuNR Biodistribution}

The PAuNRs' biodistribution was evaluated using inductively coupled plasma optical emission spectroscopy (ICP-OES) by measuring the metal concentration in the mice's harvested organs. Briefly, after day 6 post-treatment, the mice were sacrificed and harvested. The abdominal aorta blood was drawn, and the organs and tissues were collected and freeze-dried for $20 \mathrm{~h}$. Freeze-dried samples were digested in aqua regia and analysed using ICP-OES for metal concentration.

\subsection{Histological Analysis}

To prepare samples for histological analysis, the harvested organs were fixed in 10\% formaldehyde in PBS (Rapid Fixative, Kojima Chemical Industry, Saitama, Japan) for 4 days at $4{ }^{\circ} \mathrm{C}$, followed by dehydration, and, lastly, they were embedded in paraffin. The paraffin-embedded organs were sliced into $2-4 \mu \mathrm{m}$ sections. Immunohistochemistry (IHC) staining for anti-CD 31 was carried out using an automated staining processor (Discovery XT, Ventana Medical Systems, Inc., Oro Valley, AZ, USA). The vascular endothelial cells' IHC staining was carried out in pre-diluted polyclonal goat anti-CD31 antibody (1/100 dilution; sc-1506, Santa Cruz Biotechnology, Inc., Dallas, TX, USA; 2 h at room temperature) combined with a biotinylated anti-goat IgG $(\mathrm{H}+\mathrm{L})$ (BA5000, Vector Laboratories, Burlingame, CA, USA; 20 min at room temperature). Polyclonal rabbit anti-LYVE-1 antibody diluted in 1:250 (103-PA50AG, ReliaTech GmbH, Wolfenbüttel, Germany; 2 h at room temperature) combined with a biotinylated anti-rabbit IgG $(\mathrm{H}+\mathrm{L})$ (BA1000, Vector Laboratories, Burlingame, CA, USA; 20 min at room temperature) was used to carry out the IHC staining of vascular endothelial cells. A BX-51 Olympus microscope connected to a digital camera (DP72; Olympus corporation, Tokyo, Japan) was used to determine the specimen boundary at low magnification.

\subsection{Statistical Analysis}

All measurements are presented as the mean \pm SEM. Differences between any two groups were determined by one-way ANOVA followed by the Mann-Whitney test. A $p$ value $<0.05$ was considered to represent a minimal statistically significant level. Box and whisker plots displaying the first, second (median), and third quartiles were used to represent the data. Both ends of the whiskers represent the maximum and minimum values of all data. 


\section{Results}

\subsection{Synthesis and Characterisation of PAuNRs}

AuNRs with an aspect ratio of 6.71 were fabricated according to a previously reported protocol (see Materials and Methods) [34]. Many reports on the toxicity of AuNRs have been presented due to hexadecyltrimethylammonium bromide (CTAB), the surfactant used for the fabrication $[11,18,36]$. Therefore, to improve the biocompatibility of AuNRs, ligand exchange of CTAB with polyethylene glycol (PEG) was employed. The PAuNRs showed a slight red shift in LSPR by $3 \mathrm{~nm}$ after ligand exchange (Figure 1A). The TEM image of PAuNRs in Figure 1B shows rod-shaped NPs with an average length of $92.9 \mathrm{~nm}$ and width of $6.1 \mathrm{~nm}$. The zeta-potential of AuNRs after ligand exchange with PEG changed from $+42.01 \mathrm{mV}$ to $-5.27 \mathrm{mV}$ (Figure 1C). This change in the surface charge is attributed to the successful ligand exchange of the CTAB-capped AuNRs with the PEG molecule coating. The obtained PAuNRs' size and charge make them an ideal material for delivery via a lymphatic system because they can be efficiently absorbed into the lymphatic vessels [37].
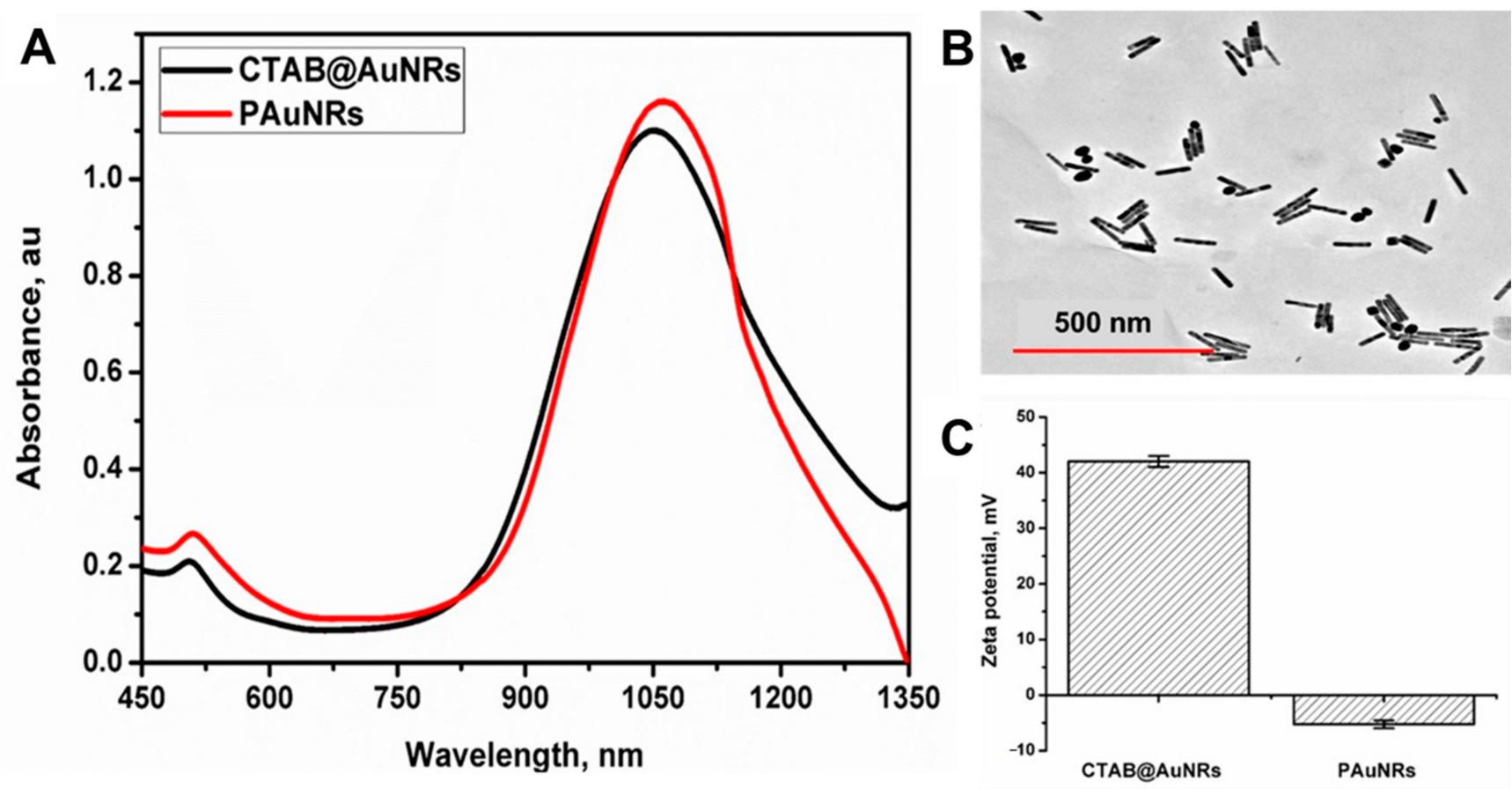

Figure 1. (A) Absorption spectra of AuNRs and PAuNRs. (B) TEM image of AuNRs (scale bar = $500 \mathrm{~nm}$ ). (C) Surface charge (zeta-potential) of AuNRs and PAuNRs (mean $\pm \mathrm{SD}, n=3$ ).

\subsection{In Vivo Time-Mediated Anti-Tumour Therapy Evaluation}

PAuNRs have been reported to accumulate efficiently in solid tumours due to passive targeting attributed to the EPR effect. The PEG polymer offers increased circulation time in the bloodstream (half-life $17 \mathrm{~h}$ ) and improved biodistribution using mice models [38,39]. We anticipate that enhanced circulation and accumulation in the metastatic environment can be obtained using the lymphatic delivery route. Since the PAuNRs' accumulation and retention in the metastatic LN can be enhanced by using the lymphatic delivery route, we proceeded to evaluate and monitor their in vivo anti-tumour activity as a function of time. Prototype mice were created using MXH10/Mo/lpr mice with unique peripheral LNs growing up to $10 \mathrm{~mm}$ in size, which is equivalent to humans. In 24 mice, FM3A/Luc cells were injected intranodally into the mice's SiLN to induce tumour metastasis in the PALN. Mice were divided into four groups: group I consisted of mice injected with PBS at AALN without laser irradiation as a control group, group II consisted of mice injected with PAuNRs at AALN without laser irradiation, group III consisted of mice injected with PBS at AALN followed by laser irradiation at PALN on day $0,1,2$, and 3 , and the last group of 
mice were injected with PAuNRs at AALN followed by laser irradiation at PALN on day 0 , 1,2 , and 3 (group IV). It is worth noting that the CTAB-capped AuNRs were not used in these experiments because of the toxicity that may arise from the CTAB [24]. IVIS Lumina was used to monitor the luciferase-expressing enzyme found in the cell activity, which enabled us to monitor the tumour growth in the SiLN as well as confirm metastasis in the PALN. The growth progression of the tumours in the SiLN and the formation of metastasis in the PALN were monitored every 3 days (Figure 2A). Metastasis in the downstream PALN of the mice started to develop after 2 weeks post-inoculation, as tumour cells continued to flow into the capsule from SiLN. This was characterised by increased luciferase activity in the PALN of each group after inoculation. Furthermore, 3D high-frequency ultrasound (VEVO-770) was used to measure the changes in the volume of the PALN. There was no significant difference in the volume changes of the PALN of each group by the end of 18 days after inoculation (Figure 2B). These results confirm the establishment of metastasis in the PALN $\left(\sim 5 \times 10^{5}\right.$ photons $\left./ \mathrm{s}\right)$. PAuNRs $(120 \mu \mathrm{L}$ of $40 \mu \mathrm{g} / \mathrm{mL})$ and phosphate-buffered saline (PBS of $120 \mu \mathrm{L}$ ) were administered to mice of each group on day 0 and day 2 from the AALN after metastasis was confirmed in the PALN.
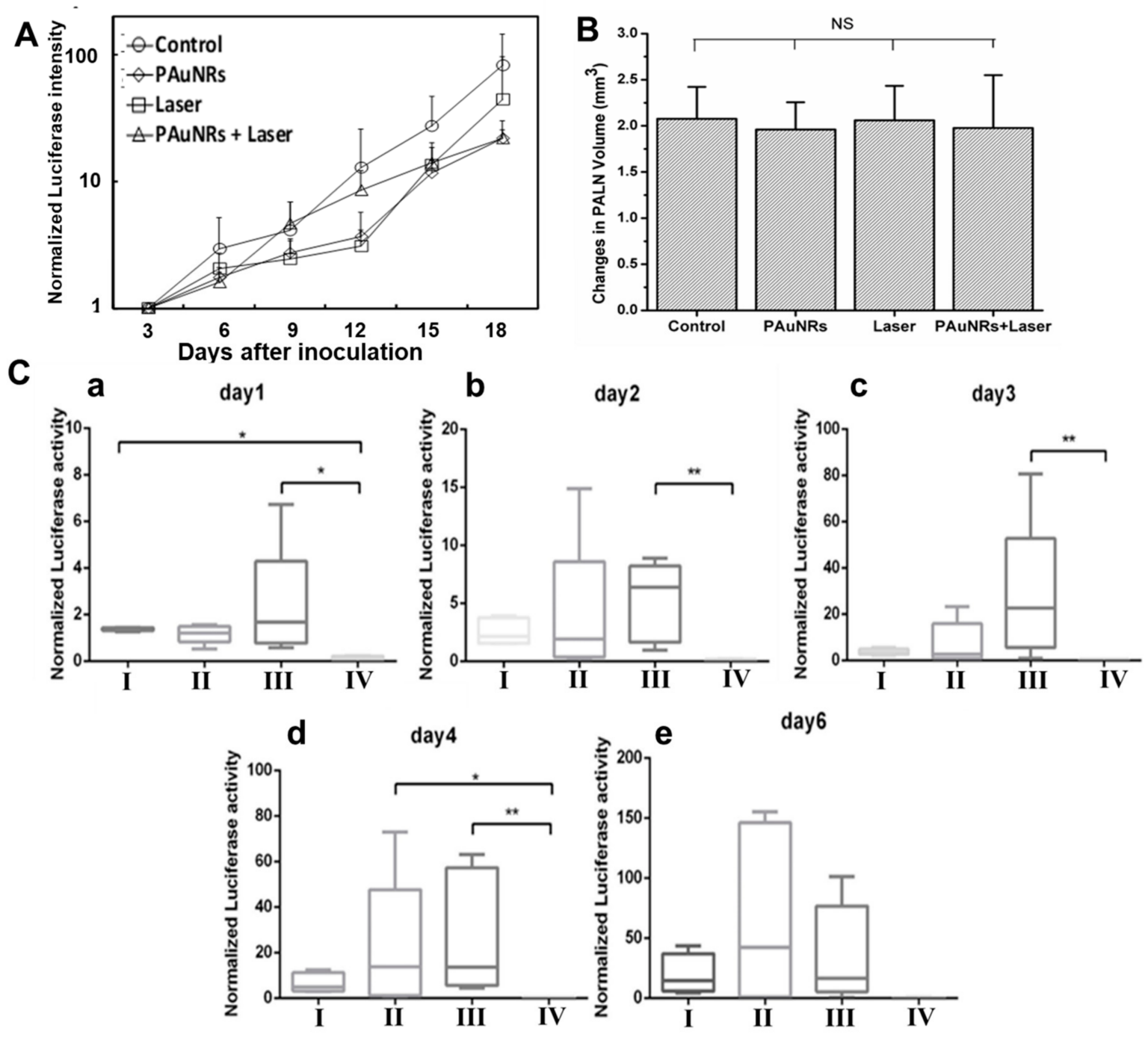

Figure 2. Confirmation of metastasis in the PALN $(n=6)$. (A) Normalised luciferase intensity in PALN measured after tumour cell inoculation, and (B) PALN volume changes measured before tumour cell inoculation and after metastasis was confirmed. (C) Illustration of normalised luciferase intensity in PALN measured after treatment $(n=6)$. (a) day 1, (b) day 2 , (c) day 3, (d) day 4, (e) day 6. NS (Not significant), ${ }^{*} p<0.05,{ }^{* *} p<0.01$. Error bars represent mean \pm SEM. 
Laser irradiation of metastatic PALN commenced $160 \mathrm{~s}$ after PAuNR injection in the AALN. The metastatic PALN was then irradiated with an NIR laser for $300 \mathrm{~s}$ after the LN surface was water-cooled to $<15{ }^{\circ} \mathrm{C}$ to prevent skin damage. The tumour activities of the mice in all groups were observed daily and then compared with each other. The optical changes related to the luciferase activities of tumour cells presented by in vivo bioluminescence imaging afforded us the opportunity to monitor treatment progress in real time. The variability in the treatment effect of each group was considered for further statistical analysis by normalising the luciferase intensities in the PALNs to the background. The tumours of group IV immediately showed negligible luciferase activity from day 1 when compared with the other groups. Smaller areas of luciferase activity were observed in groups I and group II, indicating similar tumoural activities. For the laser group, which was group III, the luciferase activity increased to the maximum, with $75 \%$ of mice showing tumour activity below 5 (Figure 2(Ca)). In group IV, tumour activity was reduced significantly as early as day $1\left({ }^{*} p<0.05\right)$. This demonstrates the immediate anti-tumour effect of the combined PAuNRs with the laser. On day 2, fresh injections of PAuNRs and PBS were performed in the groups to start the next round of treatment. Tumour activity in the control, PAuNR, and laser groups increased compared to day 1. Group II had a lower median, indicating that $50 \%$ of mice had lower tumour activity, yet $50 \%$ showed a $2-15 \%$ tumour growth increase. Although the laser group's (group III) maximum and minimum luciferase intensity were less than those of the PAuNR group (group II), the whiskers suggest similar tumour behaviour, with roughly 50\% of mice showing higher tumour activity when compared to day 1. The PAuNRs + laser group (group IV) showed a similar downward trend of reduced tumour activity compared to the other groups. The statistical difference of ${ }^{* *} p<0.01$ indicates a remarkable improvement, suggesting a further disruption of tumour angiogenesis and proliferation. Here, a $\sim 100 \%$ tumour regression result from all mice receiving metastasis treatment in this group was achieved. Figure $2(\mathrm{Cb})$ shows the result on day 2, which suggests that both group II and III did not display any therapeutic effect. Continuous tumour proliferation was observed in groups I, II, and III on day 3. Among these three groups, group III demonstrated a rapid luciferase increase despite laser irradiation. Around $50 \%$ of the mice in this group showed luciferase activity above 20, which was significantly higher than the previous day. A similar increase was also observed in group II. In contrast, group IV continued to demonstrate an efficient therapeutic effect based on its negligible luciferase intensity on day 3 (Figure 2(Cc)). A statistical difference of ${ }^{* *} p<0.01$ indicated a marked contrast in anti-tumour effect when compared to the other groups. Groups I, II, and III's tumour luciferase activity continued to increase on day 4 (Figure 2(Cd)). Group II's luciferase activity showed a rapid increase each day, with the maximum values increasing from 24 on day 3 to 76 on day 4 , and $50 \%$ of mice having between 15 and 76 luciferase intensity. However, the maximum luciferase intensity value for group III decreased from 80 on day 3 to 60 on day 4, indicating some anti-tumour effect. The median reduced from 22 on day 3 to 14 on day 4 (50\% of mice on both days). Nonetheless, this was not significant enough to cause a disruption in tumour cell growth within the metastatic area. The near-negative luciferase intensity further emphasised the role of hyperthermia-induced cell death caused by PAuNRs under laser irradiation in group IV. Compared with the other groups, ${ }^{*} p$ values of 0.05 and 0.01 were considered statistically significant differences. After a one-day interval, the therapeutic effects of the protocol were evaluated to determine tumour dynamics after day 6 . We discovered aggressive tumour activity in all the groups except group IV (Figure 2(Ce)). The maximum luciferase intensities of the control, PAuNRs, and laser group increased with the lower shifting of the median in the box. However, the medians were higher than the values obtained on day 4 , and $75 \%$ of all the mice in these groups showed continuous tumour proliferation after treatment. We attribute this occurrence to the fact that tumour activities in the PALN of mice are a function of several factors, including the immune response to the tumour, body conditions, etc. Unlike other groups, negative luciferase intensity characterised the tumour 
growth activity in group IV, signifying complete tumour destruction long after treatment ended on day 3.

Significant metastasis progression within the groups was also monitored using the intensity of light emission from the luciferase enzyme expression of tumour cells via IVIS. Figure 3A shows that in groups I, II, and III, light intensity increased from the PALN region as the treatment time continued. The photon flux density in the red region gradually expanded in size, signifying the intense luciferase activity of infiltrating tumour cells within the cortex of the lymph nodes. As the primary tumour grew, the lymphatic vessels further increased in size, allowing tumour cells to flow into the PALN, causing a partial replacement of lymphocytes within the lymph nodes [14]. This replacement thus led to a noticeable increase in the PALN size, as shown in the ultrasound image in Figure 3B. However, an immediate treatment effect was noticed in group IV after day 0 by the disappearance of luminescence light and was not visible up to the final day of treatment. This result confirms the usefulness of our proposed short-term lymphatic administration and sufficient drug accumulation for the metastatic lymph node treatment.

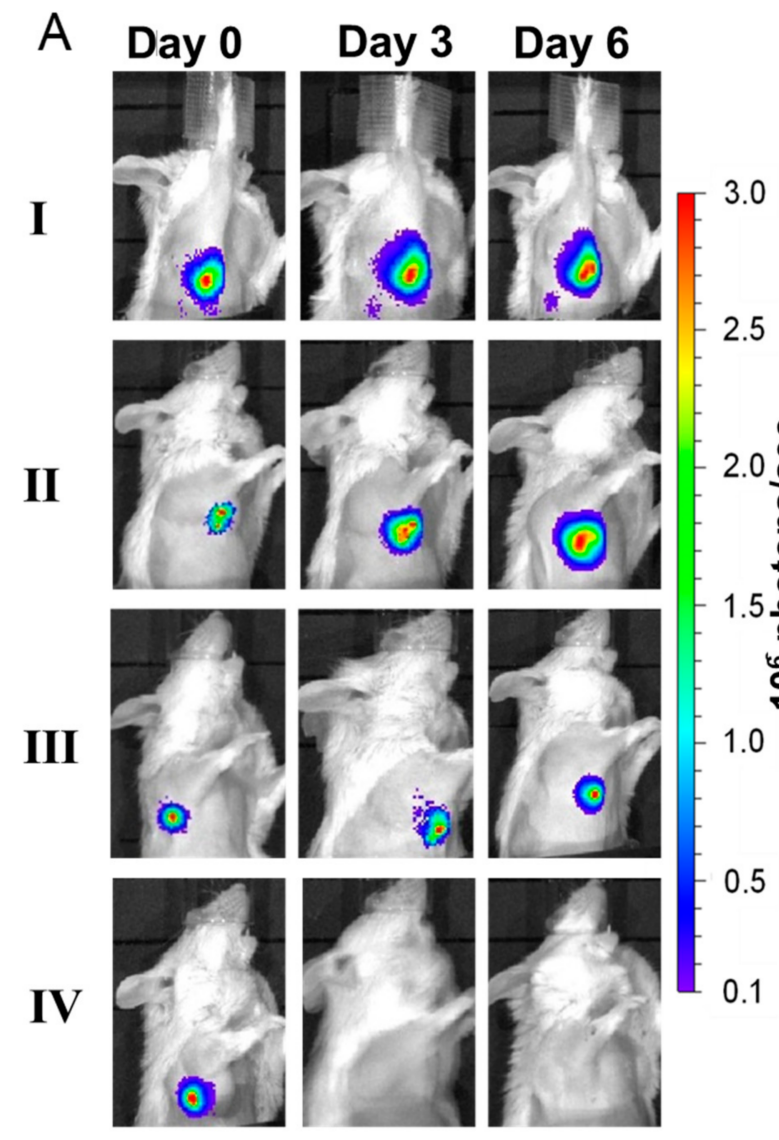

B PAuNRs PAuNRs+ Laser

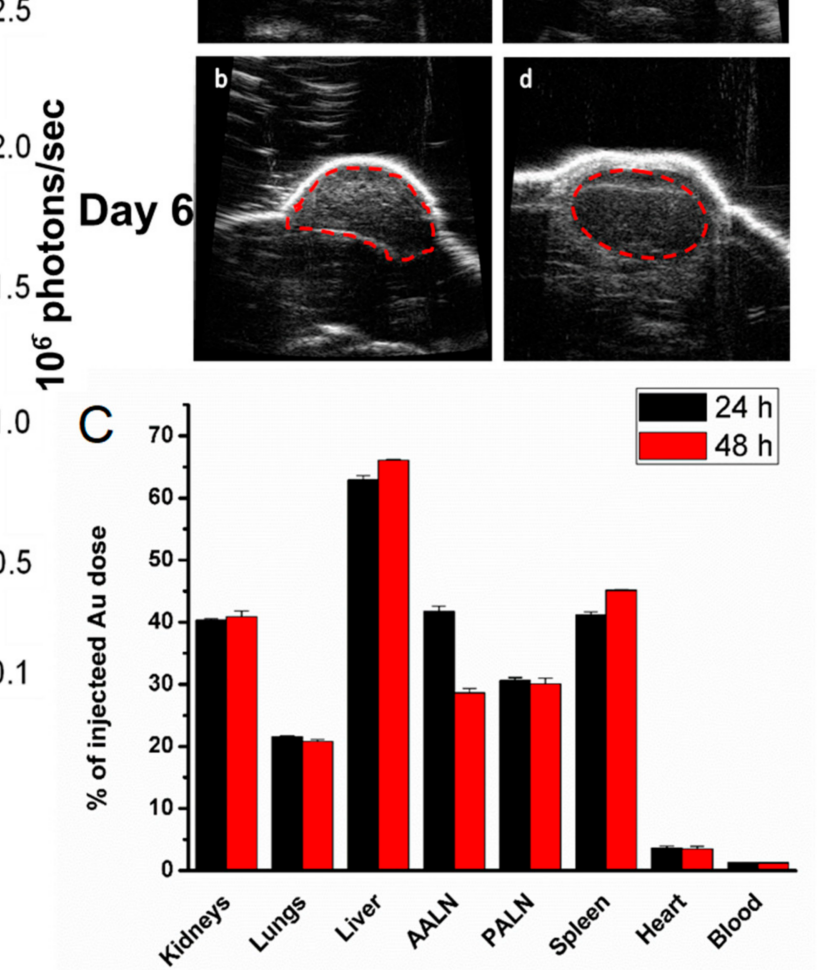

Figure 3. Anti-tumour evaluation of the treatment. (A) Representative images of tumour activities in the PALN of mice detected using an in vivo bioluminescence imaging system (IVIS) from day 0 to day 6 after treatment $(n=6)$. (B) Temporal changes in the lymph node size of PAuNRs and PAuNRs + laser groups using two-dimensional (2D) high-frequency ultrasound images. Dashed lines indicate PALN regions. (C) Biodistribution of PAuNRs in the tissues measured as Au content using ICP-OES $(n=3)$.

To assess the biodistribution kinetics of PAuNRs in vivo after lymphatic administration, we analysed the Au elemental content in tissues by inductively coupled plasma optical 
emission spectrometry (ICP-OES) at $24 \mathrm{~h}$ and $48 \mathrm{~h}$ post-administration $(120 \mu \mathrm{L}, 40 \mu \mathrm{g} / \mathrm{mL})$. The result shows a significant accumulation of PAuNRs in the PALN at $24 \mathrm{~h}$, which was maintained even after $48 \mathrm{~h}$ post-injection (Figure $3 \mathrm{C}$ ). In addition, the reticuloendothelial system (liver and spleen) retained the highest accumulation at both 24 and $48 \mathrm{~h}$ after injection, while a significant concentration reduction was observed in the other organs.

Since tumour growth occurs mainly by angiogenesis (tumours forming their own blood vessels for supply) [40], we further monitored the effectiveness of this treatment protocol by immunohistological analysis. Figure 4 shows the representative images of the treated PALN stained with anti-CD31 antibody, which distinguishes between the tumour blood vessel area and non-tumour area. In the PALN region for group IV, they were much smaller in density compared to the other two groups (II and III groups). The enlarged open lumen seen in group II and III might be caused by the metal nanoparticles and laser when used separately, as they have been shown to cause cell proliferation [24]. This was observed earlier when analysing luciferase intensity using IVIS and can be attributed to the expanded lymphatic vessels caused by rapid tumour growth in the PALN. The observed tumour blood vessel changes in group IV indicate the tumour network vessels' disruption by combining PAuNRs with laser-induced hyperthermia effects (Figure 4D,H). This inhibited the supply of nutrients to the tumour vasculature and prevented the tumour's growth capability. This result further confirms the effectiveness of the lymphatically administered PAuNRs in causing thermal damage to tumour cells and inhibiting their proliferation.
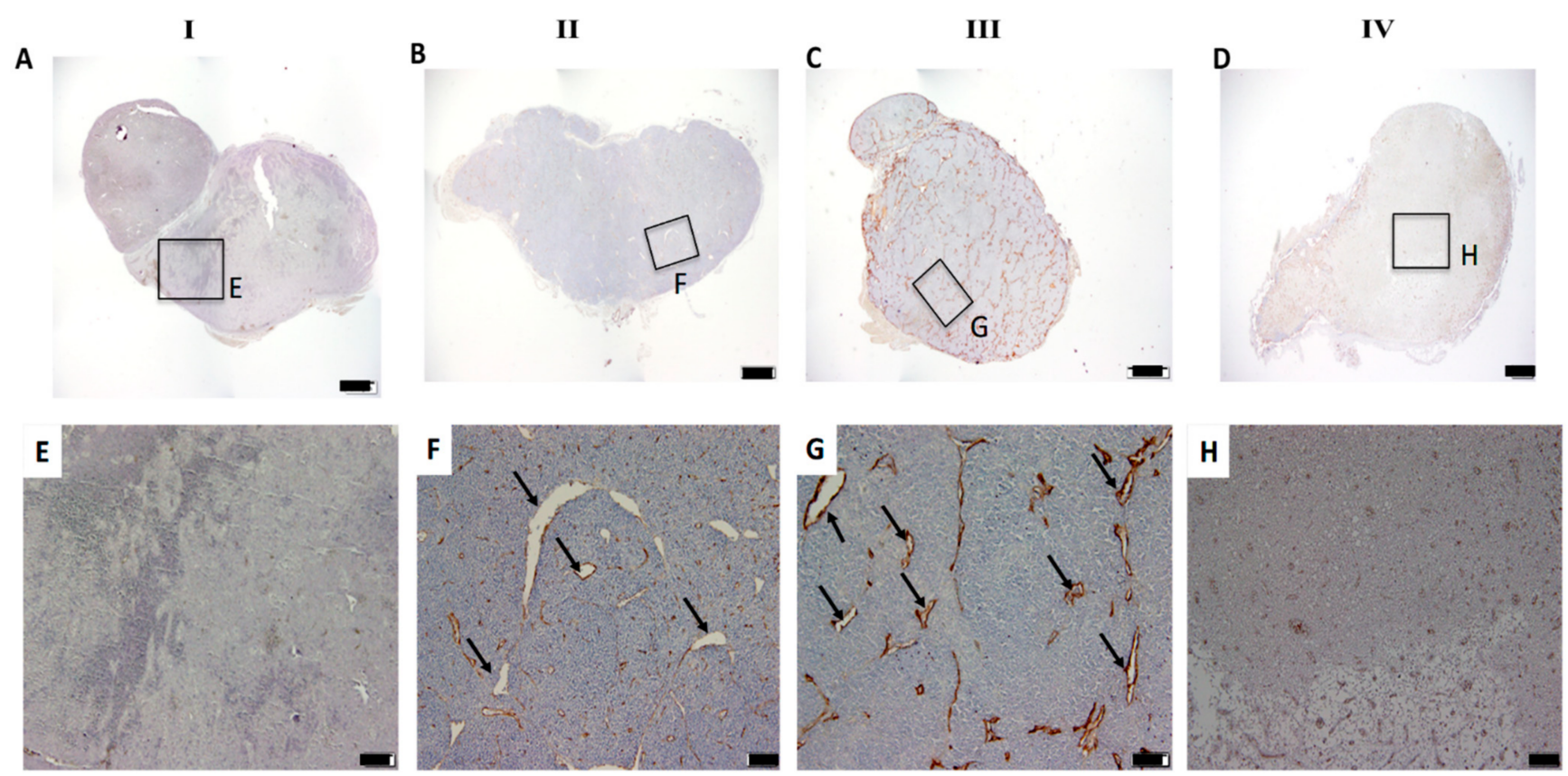

Figure 4. Histological analysis of PALN for anti-tumour effects. Representative section showing immunostaining with anti-CD31 antibody in the whole PALNs of (A) control group with PBS only, (B) control group with PAuNRs only, (C) group with PBS + laser and (D) group with PAuNRs + laser after treatment. Scale bars: $200 \mu \mathrm{m}$. Magnified view of (E) control group with PBS only, (F) control group with PAuNRs only, (G) group with PBS + laser and (H) group with PAuNRs + laser after treatment that is outlined in boxes. Scale bars: $100 \mu \mathrm{m}$; I, control group with PBS only; II, control group with PAuNRs only; III, group with PBS + laser; IV, group with PAuNRs + laser; black arrows pointing at the open lumen.

Comparatively, there was a significant difference in the therapeutic outcomes between the groups during the treatment period for the control groups (PBS and PAuNRs). The PAuNRs and laser groups showed increased luciferase activities, to a similar degree, at the end of the treatment period. The time course effect of the therapy differed significantly within the groups, with a faster therapeutic effect in the PAuNRs + laser group. In this regard, our results reveal that if the therapeutic effect is due to the first treatment only, the 
additional treatments administered should not alter the course of treatment evolution with regard to time and response rate. We have also shown that the responsiveness of aggressive metastatic cells within the PALN to therapeutic effects could be due to the frequency of treatment administration.

\section{Conclusions}

In summary, we have reported the therapeutic effect of second near-infrared absorbing gold nanorods on metastatic lymph nodes delivered via a lymphatic delivery system in a mouse model under laser irradiation. The results showed that tumour growth was completely inhibited in all mice treated with PAuNRs under laser irradiation, and the findings were statistically significant $\left({ }^{* *} p<0.01\right)$ even after treatment was concluded on day 3. In addition, the metastasis treatment through the lymphatic route was timedependent, with a significant treatment response. Furthermore, the therapeutic effect could be monitored and quantified accurately using the in vivo bioluminescence imaging system. The frequency of administering the anti-cancer agent or laser irradiation did not result in any improvement in the treatment procedure except for the laser irradiation-induced hyperthermia effect in group IV, indicating the promising potential of this protocol for the preclinical monitoring of treatment outcomes for future therapeutics.

Author Contributions: Conceptualisation, resources, and methodology, O.S.O. and T.K.; software and validation, S.M.; formal analysis and investigation, A.O.O., N.T., S.P. and O.S.O.; data curation, A.O.O. and S.M.; writing — original draft preparation, A.O.O. and O.S.O.; writing-review, editing and visualisation, O.S.O., T.C.L., N.T. and V.N.; supervision, O.S.O. and T.K.; project administration, O.S.O., S.P.S. and T.K.; funding acquisition, O.S.O., S.P.S., S.M. and T.K. All authors have read and agreed to the published version of the manuscript.

Funding: This project was supported by the South Africa / Japan Bilateral Research Program (grant no: 97982), South Africa National Research Foundation (NRF) Nanotechnology Flagship Programme (grant no: 97983), and JSPS KAKENHI grant no. 26293425 (S.M.), 16K15816 (S.M.), 26242051 (T.K.) and 24650286 (T.K.). The authors are also very grateful to the Japan Student Service Organization (JASSO) and NRF South Africa for equipment-related travel and training grants (grant no: 98299); the Competitive Program for Rated Researchers (Grant no: 106060 and 129290), Freestanding Doctoral Scholarship (Grant no: 112867 and 131237) and the University of Johannesburg, South Africa, research committee (URC) and Faculty of Science research committee (FRC) for their financial support and for the opportunity given to A.O.O., T.C.L., V.N. and N.T. to carry out their research work at the Laboratory of Biomedical Engineering for Cancer, Graduate School of Biomedical Engineering, Tohoku University, Sendai, Japan.

Institutional Review Board Statement: Animal experiments were carried out in accordance with the approved guidelines set out by the Institutional Animal Care and Use Committee of Tohoku University (Protocol: 2013BeLMO-001 \& 2013BeLMO-002 for Regulations on Genetic Recombination Experiment Safety Management and 2013BeA-019 \& 2016BeA-004 for Regulations on Animal Experimentation).

Informed Consent Statement: Not applicable.

Data Availability Statement: Data available on request due to restrictions.

Conflicts of Interest: The authors declare no conflict of interest.

\section{References}

1. Zhang, M.; Wang, J.; Shi, W.; Chen, W.; Li, W.; Shu, Y.; Liu, P.; Lu, K. Prognostic significance of metastatic lymph nodes ratio in patients with gastric adenocarcinoma after curative gastrectomy. Chin. Med. J. 2014, 127, 1874-1878.

2. Matsuki, D.; Adewale, O.; Horie, S.; Okajima, J.; Komiya, A.; Oluwafemi, O.; Maruyama, S.; Mori, S.; Kodama, T. Treatment of tumor in lymph nodes using near-infrared laser light-activated thermosensitive liposome-encapsulated doxorubicin and gold nanorods. J. Biophotonics 2017, 10, 1676-1682. [CrossRef] [PubMed]

3. Sugiura, T.; Matsuki, D.; Okajima, J.; Komiya, A.; Mori, S.; Maruyama, S.; Kodama, T. Photothermal therapy of tumors in lymph nodes using gold nanorods and near-infrared laser light with controlled surface cooling. Nano Res. 2015, 8, 3842-3852. [CrossRef] 
4. Ryan, G.M.; Kaminskas, L.M.; Porter, C.J.H. Nano-chemotherapeutics: Maximising lymphatic drug exposure to improve the treatment of lymph-metastatic cancers. J. Control. Release 2014, 193, 241-256. [CrossRef] [PubMed]

5. Kato, S.; Shirai, Y.; Motozono, C.; Kanzaki, H.; Mori, S.; Kodama, T. In vivo delivery of an exogenous molecule into murine T lymphocytes using a lymphatic drug delivery system combined with sonoporation. Biochem. Biophys. Res. Commun. 2020, 525, 1025-1031. [CrossRef] [PubMed]

6. Kato, S.; Yoshiba, S.; Mori, S.; Kodama, T. Optimization of the delivery of molecules into lymph nodes using a lymphatic drug delivery system with ultrasound. Int. J. Pharm. 2021, 597, 120324. [CrossRef]

7. Sukhbaatar, A.; Mori, S.; Saiki, Y.; Takahashi, T.; Horii, A.; Kodama, T. Lymph node resection induces the activation of tumor cells in the lungs. Cancer Sci. 2019, 110, 509-518. [CrossRef] [PubMed]

8. Stephen, Z.R.; Zhang, M. Recent Progress in the Synergistic Combination of Nanoparticle-Mediated Hyperthermia and Immunotherapy for Treatment of Cancer. Adv. Healthc. Mater. 2021, 10, 2001415. [CrossRef]

9. Zhang, Y.; He, X.; Zhang, Y.; Zhao, Y.; Lu, S.; Peng, Y.; Lu, L.; Hu, X.; Zhan, M. Native Mitochondria-Targeting polymeric nanoparticles for mild photothermal therapy rationally potentiated with immune checkpoints blockade to inhibit tumor recurrence and metastasis. Chem. Eng. J. 2021, 424, 130171. [CrossRef]

10. Park, J.E.; Kim, M.; Hwang, J.H.; Nam, J.M. Golden Opportunities: Plasmonic Gold Nanostructures for Biomedical Applications based on the Second Near-Infrared Window. Small Methods 2017, 1, 1600032. [CrossRef]

11. Yang, H.; He, H.; Tong, Z.; Xia, H.; Mao, Z.; Gao, C. The impact of size and surface ligand of gold nanorods on liver cancer accumulation and photothermal therapy in the second near-infrared window. J. Colloid Interface Sci. 2020, 565, 186-196. [CrossRef]

12. Tang, H.; Xu, X.; Chen, Y.; Xin, H.; Wan, T.; Li, B.; Pan, H.; Li, D.; Ping, Y. Reprogramming the Tumor Microenvironment through Second-Near-Infrared-Window Photothermal Genome Editing of PD-L1 Mediated by Supramolecular Gold Nanorods for Enhanced Cancer Immunotherapy. Adv. Mater. 2021, 33, 2006003. [CrossRef] [PubMed]

13. Austin, L.A.; Mackey, M.A.; Dreaden, E.C.; El-Sayed, M.A. The optical, photothermal, and facile surface chemical properties of gold and silver nanoparticles in biodiagnostics, therapy, and drug delivery. Arch. Toxicol. 2014, 88, 1391-1417. [CrossRef] [PubMed]

14. Cong, B. Gold nanorods: Near-infrared plasmonic photothermal conversion and surface coating. J. Mater. Sci. Chem. Eng. 2014, 2, 20. [CrossRef]

15. Lebepe, T.C.; Parani, S.; Oluwafemi, O.S. Graphene Oxide-Coated Gold Nanorods: Synthesis and Applications. Nanomaterials 2020, 10, 2149. [CrossRef]

16. Centi, S.; Tatini, F.; Ratto, F.; Gnerucci, A.; Mercatelli, R.; Romano, G.; Landini, I.; Nobili, S.; Ravalli, A.; Marrazza, G. In vitro assessment of antibody-conjugated gold nanorods for systemic injections. J. Nanobiotechnol. 2014, 12, 55. [CrossRef] [PubMed]

17. Liopo, A.V.; Conjusteau, A.; Oraevsky, A.A. PEG-coated gold nanorod monoclonal antibody conjugates in preclinical research with optoacoustic tomography, photothermal therapy, and sensing. In Photons Plus Ultrasound: Imaging and Sensing 2012; Oraevsky, A.A., Wang, L.V., Eds.; SPIE: Washington, DC, USA, 2012; p. 822344.

18. Liu, K.; Zheng, Y.; Lu, X.; Thai, T.; Lee, N.A.; Bach, U.; Gooding, J.J. Biocompatible gold nanorods: One-step surface functionalization, highly colloidal stability, and low cytotoxicity. Langmuir 2015, 31, 4973-4980. [CrossRef] [PubMed]

19. Tebbe, M.; Kuttner, C.; Männel, M.; Fery, A.; Chanana, M. Colloidally stable and surfactant-free protein-coated gold nanorods in biological media. ACS Appl. Mater. Interfaces 2015, 7, 5984-5991. [CrossRef]

20. Yasun, E.; Li, C.; Barut, I.; Janvier, D.; Qiu, L.; Cui, C.; Tan, W. BSA modification to reduce CTAB induced nonspecificity and cytotoxicity of aptamer-conjugated gold nanorods. Nanoscale 2015, 7, 10240-10248. [CrossRef]

21. Brannon-Peppas, L.; Blanchette, J.O. Nanoparticle and targeted systems for cancer therapy. Adv. Drug Deliv. Rev. 2004, 56, 1649-1659. [CrossRef]

22. Aioub, M.; El-Sayed, M.A. A real-time surface enhanced raman spectroscopy study of plasmonic photothermal cell death using targeted gold nanoparticles. J. Am. Chem. Soc. 2016, 138, 1258-1264. [CrossRef]

23. De Freitas, L.; Zanelatto, L.; Mantovani, M.; Silva, P.; Ceccini, R.; Grecco, C.; Moriyama, L.; Kurachi, C.; Martins, V.; Plepis, A. In Vivo photothermal tumour ablation using gold nanorods. Laser Phys. 2013, 23, 066003. [CrossRef]

24. Oladipo, A.O.; Oluwafemi, O.S.; Songca, S.P.; Sukhbaatar, A.; Mori, S.; Okajima, J.; Komiya, A.; Maruyama, S.; Kodama, T. A novel treatment for metastatic lymph nodes using lymphatic delivery and photothermal therapy. Sci. Rep. 2017, 7, 45459. [CrossRef]

25. Kato, S.; Mori, S.; Kodama, T. A novel treatment method for lymph node metastasis using a lymphatic drug delivery system with nano/microbubbles and ultrasound. J. Cancer 2015, 6, 1282. [CrossRef] [PubMed]

26. Turner, P.V.; Brabb, T.; Pekow, C.; Vasbinder, M.A. Administration of substances to laboratory animals: Routes of administration and factors to consider. J. Am. Assoc. Lab. Anim. Sci. 2011, 50, 600-613. [PubMed]

27. Sato, T.; Mori, S.; Arai, Y.; Kodama, T. The combination of intralymphatic chemotherapy with ultrasound and nano-/microbubbles is efficient in the treatment of experimental tumors in mouse lymph nodes. Ultrasound Med. Biol. 2014, 40, 1237-1249. [CrossRef] [PubMed]

28. Kikuchi, R.; Sukhbaatar, A.; Sakamoto, M.; Mori, S.; Kodama, T. A model system for studying superselective radiotherapy of lymph node metastasis in mice with swollen lymph nodes. Clin. Transl. Radiat. Oncol. 2020, 20, 53-57. [CrossRef]

29. Kodama, T.; Hatakeyama, Y.; Kato, S.; Mori, S. Visualization of fluid drainage pathways in lymphatic vessels and lymph nodes using a mouse model to test a lymphatic drug delivery system. Biomed. Opt. Express. 2015, 6, 124-134. [CrossRef] 
30. Fukumura, R.; Sukhbaatar, A.; Mishra, R.; Sakamoto, M.; Mori, S.; Kodama, T. Study of the physicochemical properties of drugs suitable for administration using a lymphatic drug delivery system. Cancer Sci. 2021, 112, 1735. [CrossRef]

31. Kodama, T.; Matsuki, D.; Tada, A.; Takeda, K.; Mori, S. New concept for the prevention and treatment of metastatic lymph nodes using chemotherapy administered via the lymphatic network. Sci. Rep. 2016, 6, 32506. [CrossRef]

32. Shen, K.-H.; Lu, C.-H.; Kuo, C.-Y.; Li, P.-Y.; Yeh, Y.-C. Smart near infrared-responsive nanocomposite hydrogels for therapeutics and diagnostics. J. Mater. Chem. B 2021. [CrossRef]

33. Shao, L.; Mori, S.; Yagishita, Y.; Okuno, T.; Hatakeyama, Y.; Sato, T.; Kodama, T. Lymphatic mapping of mice with systemic lymphoproliferative disorder: Usefulness as an inter-lymph node metastasis model of cancer. J. Immunol. Methods 2013, 389, 69-78. [CrossRef]

34. Ye, X.; Zheng, C.; Chen, J.; Gao, Y.; Murray, C.B. Using binary surfactant mixtures to simultaneously improve the dimensional tunability and monodispersity in the seeded growth of gold nanorods. Nano Lett. 2013, 13, 765-771. [CrossRef] [PubMed]

35. Okuno, T.; Kato, S.; Hatakeyama, Y.; Okajima, J.; Maruyama, S.; Sakamoto, M.; Mori, S.; Kodama, T. Photothermal therapy of tumors in lymph nodes using gold nanorods and near-infrared laser light. J. Control. Release 2013, 172, 879-884. [CrossRef] [PubMed]

36. Zhu, X.-M.; Fang, C.; Jia, H.; Huang, Y.; Cheng, C.H.; Ko, C.-H.; Chen, Z.; Wang, J.; Wang, Y.-X.J. Cellular uptake behaviour, photothermal therapy performance, and cytotoxicity of gold nanorods with various coatings. Nanoscale 2014, 6, 11462-11472. [CrossRef] [PubMed]

37. Peng, X.; Wang, J.; Zhou, F.; Liu, Q.; Zhang, Z. Nanoparticle-based approaches to target the lymphatic system for antitumor treatment. Cell. Mol. Life Sci. 2021, 78, 5139-5161. [CrossRef] [PubMed]

38. Niidome, T.; Ohga, A.; Akiyama, Y.; Watanabe, K.; Niidome, Y.; Mori, T.; Katayama, Y. Controlled release of PEG chain from gold nanorods: Targeted delivery to tumor. Biorg. Med. Chem. 2010, 18, 4453-4458. [CrossRef] [PubMed]

39. Ma, X.; Cheng, Y.; Huang, Y.; Tian, Y.; Wang, S.; Chen, Y. PEGylated gold nanoprisms for photothermal therapy at low laser power density. RSC Adv. 2015, 5, 81682-81688. [CrossRef]

40. Stacker, S.A.; Williams, S.P.; Karnezis, T.; Shayan, R.; Fox, S.B.; Achen, M.G. Lymphangiogenesis and lymphatic vessel remodelling in cancer. Nat. Rev. Cancer 2014, 14, 159-172. [CrossRef] 